\title{
ADESÃO DO CHEKLIST CIRÚRGICO À LUZ DA CULTURA DE SEGURANÇA DO PACIENTE
}

\author{
Surgical checklist accession in light of patient safety culture
}

Adhesión del checklist quirúrgico a la luz de la cultura de seguridad del paciente

Maíra Cássia Borges de Oliveira ${ }^{1 *}$, Amildo Korb², Denise Antunes de Azambuja Zocche ${ }^{3}$, Danielle Cabral Bezerra', Fabiane Pertille ${ }^{5}$, Jucimar Frigo ${ }^{6}$

RESUMO: Objetivo: Analisar a produção científica nacional e internacional sobre a adesão de checklist cirúrgico quanto à segurança do paciente. Método: Revisão integrativa da literatura utilizando as bases de dados Scientific Eletronic Library Online (SciELO), Literatura Latino-Americana em Ciências da Saúde (LILACS), PubMed e Scopus, no período de janeiro de 2007 a julho de 2017. Resultados: Dos 32 estudos que contemplaram o objetivo deste trabalho, 53,1\% foram publicados em língua portuguesa e 40,6\% no ano de 2015. Entre os temas analisados, destacam-se adesão ao protocolo (40,6\%), registros sobre cirurgia segura (37,5\%), elaboração e implementação da lista de verificação $(9,4 \%)$, percepção dos profissionais $(9,4 \%)$ e importância da visita pós-operatória (3,1\%). Quanto à implementação dos protocolos de cirurgia segura, 40,6\% relataram sobre educação permanente e 21,9\%, sobre comunicação. Conclusão: A utilização de checklist para cirurgia segura está sendo cada vez mais elucidada nos serviços de saúde, a partir da comunicação, buscando promover cuidado centrado no paciente.

Palavras-chave: Centros cirúrgicos. Segurança do paciente. Comunicação. Enfermagem.

ABSTRACT: Objective: To analyze the national and international scientific production on adherence to the surgical checklist regarding patient safety. Method: Integrative literature review using the following databases: Scientific Electronic Library Online (SciELO), Literatura Latino-Americana em Ciências da Saúde (LILACS), PubMed and Scopus, from January 2007 to July 2017. Results: Of the 32 studies that included the objective of this study, 53.1\% were published in Portuguese, and $40.6 \%$ in the year 2015. Among the subjects analyzed, special reference is made to protocol compliance (40.6\%), records on safe surgery (37.5\%), preparation and implementation of the checklist (9.4\%), professionals perception (9.4\%) and importance of the postoperative visit (3.1\%). Regarding the implementation of safe surgery protocols, $40.6 \%$ reported on permanent education and $21.9 \%$ on communication. Conclusion: The use of checklist for safe surgery is being increasingly elucidated in health services, using communication to to promote patient-centered care.

Keywords: Surgicenters. Patient safety. Communication. Nursing.

RESUMEN: Objetivo: Analizar la producción científica nacional e internacional sobre la adhesión de checklist quirúrgico en cuanto a la seguridad del paciente. Método: Revisión integrativa de la literatura usando las bases de datos Scientific Eletronic Library Online (SciELO), Literatura Latino-Americana em Ciências da Saúde (LILACS), PubMed y Scopus, en el período de enero de 2007 a julio de 2017. Resultados: De los 32 estudios que abarcan el objetivo de este estudio, 53,1\% fueron publicados en portugués y 40,6\% en 2015. Entre los temas analizados, se destacan adhesión al protocolo (40,6\%), registros sobre cirugía segura (37,5\%), elaboración e implementación de la lista de verificación $(9,4 \%)$, percepción de los profesionales $(9,4 \%)$ y importancia de la visita postoperatoria (3,1\%). En cuanto a la implementación de los protocolos de cirugía segura, 40,6\% relató sobre educación permanente y 21,9\%, sobre comunicación. Conclusión: La utilización de checklist para cirugía segura está siendo cada vez más elucidada en los servicios de salud, a partir de la comunicación, buscando promover cuidado centrado en el paciente. Palabras clave: Centros quirúrgicos. Seguridad del paciente. Comunicación. Enfermería.

'Discente do curso de Enfermagem da Universidade do Estado de Santa Catarina (Udesc Oeste) - Chapecó (SC), Brasil.

Biólogo; doutor em Meio Ambiente e Desenvolvimento pela Universidade Federal do Paraná (UFPR); docente do Departamento de Enfermagem da Udesc Oeste - Chapecó (SC), Brasil.

${ }^{3}$ Enfermeira; doutora em Enfermagem pela Universidade Federal do Rio Grande do Sul (UFRGS); professora adjunta do curso de Enfermagem da Udesc (campus Chapecó) - Chapecó (SC), Brasil.

"Doutora em Enfermagem pela Universidade de São Paulo; enfermeira; docente no Departamento de Enfermagem da Udesc Oeste - Chapecó (SC), Brasil.

${ }^{5}$ Enfermeira; pós-graduada em Auditoria Serviços de Saúde na Faculdade São Fidelis (FSF) e em Terapia Intensiva na Universidade do Contestado (UNC) (campus Concórdia) - Concórdia (SC). Brasil.

${ }^{6}$ Enfermeira; mestre em Terapia Intensiva pela Sociedade Brasileira de Terapia Intensiva (Sobrati) - Santo André (SP), Brasil.

*Autor correspondente: mairaacassia@gmail.com

Recebido: 08/11/2017 - Aprovado: 02/01/2018

DOI: $10.5327 / 21414-4425201800010007$ 


\section{INTRODUÇÃO}

A Organização Mundial da Saúde (OMS), em 2004, instituiu o World Alliance for Patient Safety, que tem o objetivo de organizar os conceitos e as definições acerca da segurança do paciente e, assim, propor medidas que visem à redução de danos e à implementação de listas de verificação, com foco em gestão de saúde e segurança, de modo a substituir a culpabilização pelo aprender com as falhas no processo de trabalho em saúde ${ }^{1,2}$.

No Brasil, essa temática alcançou destaque em 2013, com a elaboração do Programa Nacional de Segurança do Paciente (PNSP), que apoiou a implantação da gestão de risco e de Núcleos de Segurança do Paciente (NSS) nos estabelecimentos de saúde ${ }^{2,3}$.

Nesse contexto, a comunicação segura e efetiva entre a equipe de saúde é um fator determinante para reduzir riscos, a partir da valorização da percepção, de atitudes e do comportamento de todos os profissionais envolvidos no cuidado ao paciente, com vistas à promoção da cultura de segurança. Desta forma, a liderança participativa se faz presente na comunicação de seu processo de trabalho, em que essa equipe deve se comunicar abertamente sobre as inquietudes nas falhas de segurança, sem receio de culpabilização ou punição ${ }^{4}$. Para isso, o serviço de saúde deve estar estruturado seguramente, contando com uma adequada gestão de riscos, para utilizar tecnologias, processos e recursos humanos, uma vez que os erros e danos têm causa multifatorial ${ }^{5}$.

Frente ao exposto, é imprescindível a educação permanente na enfermagem, uma vez que estabelece cultura institucional de segurança com mudanças de hábitos e atitudes, ou seja, aprendendo e ressignificando práticas profissionais. Ademais, o enfermeiro, como gestor ou gerente, tem, entre outras atribuições, a função de coordenar as equipes e os processos de trabalho, quer sejam no âmbito hospitalar ou na atenção básica. Para isso, o enfermeiro deve treinar, capacitar e conscientizar sua equipe para prestar assistência qualificada, segura e centrada no paciente ${ }^{6}$.

\section{OBJETIVO}

Analisar a produção científica nacional e internacional sobre a adesão ao checklist cirúrgico, com vistas à implementação da cirurgia segura nos serviços hospitalares de saúde.

\section{MÉTODO}

Trata-se de uma revisão integrativa, que possibilita a construção de análise ampla da literatura escolhida, contribuindo para discussões e reflexões acerca da temática proposta. A construção desta revisão envolveu identificação do tema e formulação da questão de pesquisa, aplicação de critérios de inclusão e exclusão, definição das informações extraídas dos estudos, avaliação dos estudos incluídos, interpretação dos resultados e apresentação da síntese dos resultados ${ }^{7}$.

Para a seleção dos artigos, realizaram-se buscas nas bases de dados: Scientific Eletronic Library Online (SciELO), Literatura Latino-Americana em Ciências da Saúde (LILACS), PubMed e Scopus. Utilizou-se no estudo a terminologia em saúde encontrada nos Descritores em Ciências da Saúde (DeCS) e no Medical Subject Headings (MeSH), ambos com vocabulário que permite linguagem padrão e unificada para a indexação de estudos e periódicos científicos. Os termos "Checklist", "Guideline Adherence", "Patient Safety" e "Surgery" foram utilizados na busca dos artigos, combinados com os operadores booleanos "and" e "or": "Checklist AND Guideline Adherence OR Patient Safety"; "Patient Safety AND Checklist AND Surgery"; "Checklist AND Surgery OR Guideline Adherence".

A coleta de dados ocorreu entre julho e agosto de 2017, com a seguinte questão norteadora: como o uso do checklist direcionado para a implementação da cirurgia segura está inserido no atendimento prestado aos pacientes cirúrgicos?

Os critérios de inclusão foram os trabalhos publicados em formato de artigo na íntegra, relacionados com o tema cirurgia segura e publicados no período de janeiro de 2007 a julho de 2017. Os trabalhos incluídos foram publicados nos idiomas português, inglês e espanhol. Excluíram-se, assim, publicações como teses, dissertações, resumos e trabalhos de conclusão de curso.

Do total de 70 artigos encontrados, foram excluídos 18 estudos por não estarem na íntegra; 11 por não abordarem a temática de estudo, 8 por estarem repetidos nas bases selecionadas e 1 por ser dissertação. Portanto, das 70 publicações iniciais, 32 artigos foram utilizados como amostra deste estudo.

Para facilitar a análise dos estudos elegíveis, utilizou-se uma planilha eletrônica no Microsoft Excel ${ }^{\circledast}$ para coleta de dados, contendo ano de publicação, Qualis/ fator de impacto, país de origem, idioma, tipo de estudo, temática abordada e descritores.

Com relação aos dados obtidos segundo os descritores, foi elaborado um mapa conceitual que permitiu estruturar 
o percurso da análise dos dados, por meio da hierarquização de ideias, auxiliando, de forma efetiva, a compreensão do que estava sendo analisado ${ }^{8}$.

Para facilitar a visualização do método de busca dos estudos, elaborou-se um fluxograma do tipo Prisma ${ }^{9}$ (Figura 1).

Por se tratar de uma pesquisa do tipo revisão de literatura, não houve envolvimento direto com seres humanos, porém, convêm sinalizar que foram seguidos os preceitos éticos contidos nas normas nacionais e internacionais que regulamentam os Comitês de Ética em Pesquisa.

\section{RESULTADOS}

Dos 70 artigos analisados, 32 estudos abordaram a temática de cirurgia segura nos serviços de saúde. Desses, 13 (40,6\%) foram publicados em 2015, 8 (25,0\%) em 2014, 6 (18,5\%) em 2013, 3 (9,4\%) em 2016 e 2 (6,2\%) em 2012.

Quanto à distribuição dos periódicos, foi constatado que $19(59,40 \%)$ são nacionais, com destaque à Revista SOBECC e à Revista Latino-Americana de Enfermagem, com 4 (12,50\%) e $3(9,37 \%)$ estudos publicados, respectivamente. Quanto ao

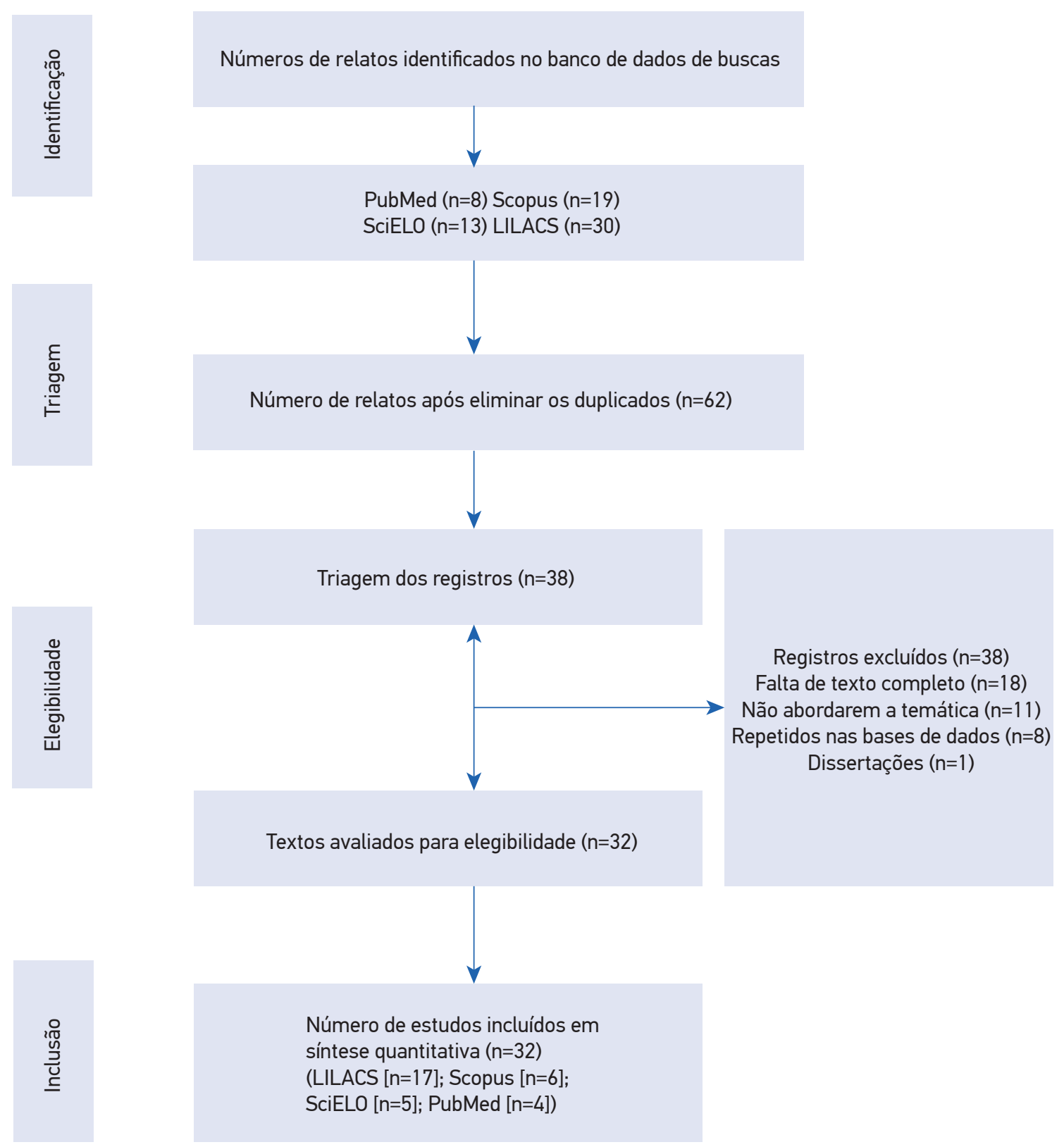

Figura 1. Fluxograma do método de busca dos estudos da revisão integrativa. 
Qualis-CAPES (Coordenação de Aperfeiçoamento de Pessoal de Nível Superior), 12 periódicos nacionais apresentam a seguinte estratificação: 1 - A1; 2 - A2; 5 - B1; 1 - B2 e 1 - B3. Sobre o fator de impacto dos periódicos, a Revista Latino-Americana de Enfermagem atribui com 0,5797, conforme Tabela 1. Em relação aos periódicos internacionais, perfazem-se 13 (40,60\%), sendo 2 estudos $(6,30 \%)$ da BMJ Open Journals, seguido de outros periódicos com $1(3,10 \%)$ publicação cada. Do total das revistas internacionais, duas são voltadas para a publicação de temas sobre cirurgias: ANZ Journal of Surgery e International
Journal of Surgery. Quanto ao Qualis para periódicos internacionais, quatro possuem estratificação distribuída da seguinte forma: 2 - B1; 1 - B2 e 1 - B3. O maior fator de impacto atribuído foi ao periódico Implementation Science, com 3,354, conforme Tabela 1.

Quanto à abordagem metodológica dos estudos, 10 $(31,25 \%)$ são de levantamento; $5(15,62 \%)$ retrospectivos; 4 (12,50\%) observacionais; 3 (9,37\%) estudos de campo; 3 $(9,37 \%)$ revisões; $3(9,37 \%)$ transversais; $1(3,12 \%)$ comparativo; 1 (3,12\%) do tipo documental; 1 (3,12\%) ensaio teórico e $1(3,12 \%)$ prospectivo.

Tabela 1. Distribuição da produção científica nacional e internacional, de acordo com o periódico, fator de impacto e classificação Qualis-CAPES.

\begin{tabular}{|c|c|c|c|}
\hline Periódicos Nacionais & n (\%) & Qualis & Fator de impacto \\
\hline Revista SOBECC & $04(12,5)$ & B3 & - \\
\hline Revista Latino-Americana de Enfermagem & $03(9,4)$ & $\mathrm{A} 1$ & 0,5797 \\
\hline Revista Acta Paulista de Enfermagem & $02(6,3)$ & A2 & 0,5083 \\
\hline Revista Gaúcha de Enfermagem & $02(6,3)$ & B1 & 0,4048 \\
\hline Escola Anna Nery Revista de Enfermagem & $01(3,1)$ & B1 & 0,3651 \\
\hline Cadernos de Saúde Pública & $01(3,1)$ & B1 & 0,4860 \\
\hline Revista da Escola de Enfermagem da USP & $01(3,1)$ & A2 & 0,4585 \\
\hline Revista de Enfermagem da UERJ & $01(3,1)$ & B1 & - \\
\hline Revista Brasileira de Ortopedia & $01(3,1)$ & B4 & 0,1667 \\
\hline Revista de Enfermagem do Centro-Oeste Mineiro & $01(3,1)$ & B2 & - \\
\hline Revista Brasileira de Anestesiologia & $01(3,1)$ & B1 & 0,0719 \\
\hline Cuidado é Fundamental Online & $01(3,1)$ & B2 & - \\
\hline Subtotal & $19(59,4)$ & & \\
\hline Periódicos Internacionais & n (\%) & Qualis & Fator de impacto \\
\hline BMJ Open Journals & $2(6,3)$ & - & 2,369 \\
\hline Revista del Hospital Aeronáutico Central & $1(3,1)$ & - & - \\
\hline Archivos Argentinos de Pediatría & $1(3,1)$ & B3 & 0,403 \\
\hline International Journal for Quality in Health Care & $1(3,1)$ & - & 2,342 \\
\hline $\begin{array}{l}\text { Scandinavian Journal of Trauma, } \\
\text { Resuscitation and Emergency Medicine }\end{array}$ & $1(3,1)$ & - & 2,036 \\
\hline Enfermería Global & $1(3,1)$ & B1 & 0,2549 \\
\hline The Journal of Laryngology \& Otology & $1(3,1)$ & - & 0,8440 \\
\hline Plos One & $1(3,1)$ & - & 2,8060 \\
\hline Implementation Science & $1(3,1)$ & - & 3,3540 \\
\hline ANZ Journal of Surgery & $1(3,1)$ & - & 1,1220 \\
\hline Journal of Infection and Public Health & $1(3,1)$ & B1 & 1,4390 \\
\hline International Journal of Surgery & $1(3,1)$ & B2 & 0,6900 \\
\hline Subtotal & $13(40,6)$ & & \\
\hline Total & $32(100,0)$ & & \\
\hline
\end{tabular}

CAPES: Coordenação de Pessoal de Nível Superior; USP: Universidade de São Paulo; UERJ: Universidade do Estado do Rio de Janeiro. 
Em relação ao idioma, 17 (53,1\%) estão em língua portuguesa, 13 (40,6\%) em língua inglesa e 2 (6,3\%) em espanhol.

Após a análise dos estudos, os temas preponderantes nessa revisão foram: adesão de protocolos voltados para a cirurgia $(40,60 \%)$, registros sobre cirurgia segura $(37,50 \%)$, elaboração e implementação de listas de verificação $(9,40 \%)$, percepção dos profissionais em relação à cirurgia segura $(9,40 \%)$ e importância da visita pós-operatória $(3,10 \%)$. Sobre a implementação de protocolos de cirurgia segura com referencial no manual "Cirurgias Seguras Salvam Vidas"10, 13 (40,60\%) abordaram a educação permanente como importante ferramenta na segurança do paciente e $7(21,90 \%)$ relataram sobre a importância da comunicação entre as equipes de saúde. Em relação às palavras-chave, os descritores mais frequentemente utilizados pelos autores dos estudos foram "segurança do paciente", em 22 (68,75\%) estudos, e "lista de checagem", utilizado em $10(31,25 \%)$ publicações.

Após análise dos descritores e do agrupamento dos temas, foi possível realizar um mapa conceitual, representado na Figura 2.

Os grupos temáticos que emergiram dos descritores oriundos das publicações foram: fatores dependentes, fatores relacionados e fatores que influenciam na incorporação de protocolos voltados para a cirurgia segura.

\section{DISCUSSÃO}

As publicações analisadas neste estudo são recentes, pois se concentraram nos últimos 5 anos (2012 a 2017). Esse fato pode ser explicado a partir da publicação das diretrizes preconizadas pela OMS, em 2008, e do PNSP, em 2013, no Brasil. Isso é uma evidência importante, tanto no cenário nacional como internacional, pois os eventos adversos (EA) nos procedimentos cirúrgicos são precedentes de lesões ou danos ao paciente, podendo ocasionar incapacidades e/ou mortes ${ }^{1,3,11}$. Além disso, a seção três do documento "Segundo Desafio Global para a Segurança do Paciente: Cirurgias Seguras Salvam Vidas", da OMS disponibiliza modelos de listas de verificação a serem utilizados nos processos de trabalho cirúrgico, podendo ser adaptados ou adequados às necessidades de cada instituição de saúde e às demandas trazidas pela equipe cirúrgica, ${ }^{1,12,13}$.

Ressalta-se que essa lista garante segurança ao paciente no momento anterior à indução anestésica (identificação/ sign in), posterior à indução e anterior à incisão cirúrgica (confirmação/timeout) e durante ou imediatamente após o fechamento da ferida (registro/sign out) ${ }^{1}$.

No Brasil, a adesão às listas de verificação vem sendo processual nos serviços de saúde, porém com alguns desafios, como a conscientização indevida da importância de seu uso pelos profissionais da equipe cirúrgica, com sinais, ainda, de culpabilização e indenizações pelas práticas inseguras ${ }^{14}$. A viabilidade da lista de verificação de segurança cirúrgica vem se apresentando promissora em diversos hospitais brasileiros, embora haja, ainda, baixo engajamento da equipe cirúrgica em sua adesão. O enfermeiro, como coordenador do setor cirúrgico, pode se apropriar dessa ferramenta como forma de mensurar e avaliar a assistência prestada.

Ademais, as habilidades médicas, a comunicação efetiva e a consciência integral da equipe cirúrgica sobre os riscos envolvidos no ato anestésico-cirúrgico qualificam o processo e os resultados na terapêutica segura e efetiva ao paciente cirúrgico.

Assim, faz-se necessária a presença do coordenador da lista para verificar, verbalmente, com o paciente a sua identidade, o sítio da cirurgia, o procedimento e o consentimento obtido $^{12}$. O coordenador confirmará visualmente se o sítio

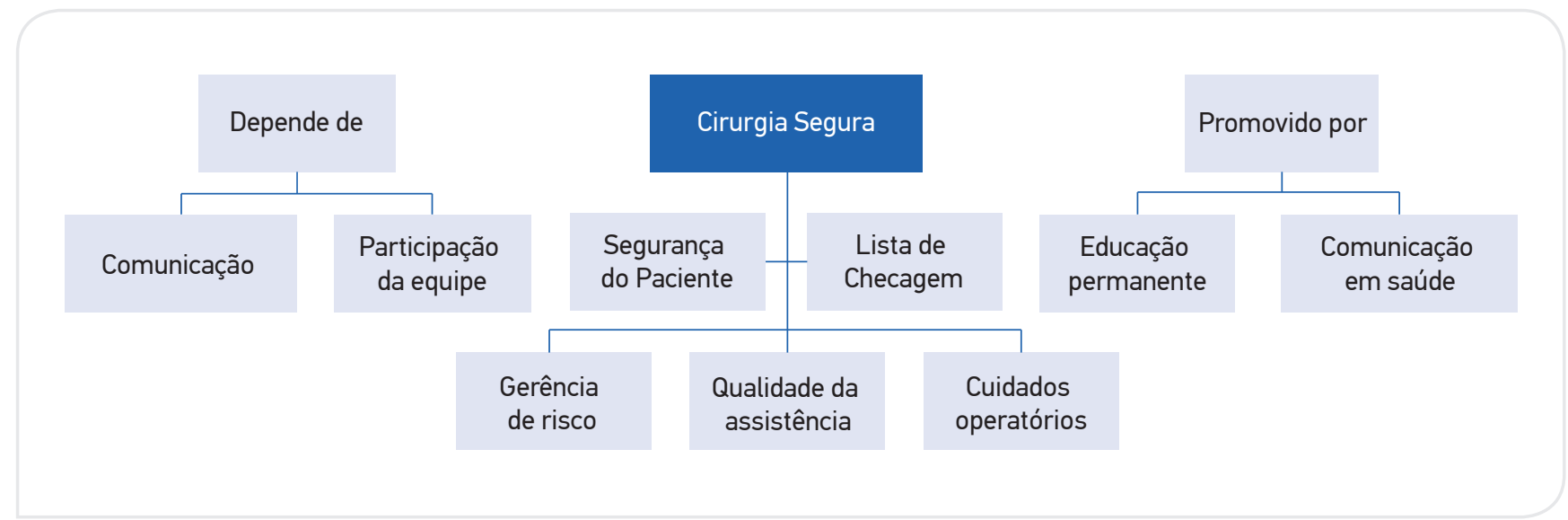

Figura 2. Mapa conceitual elaborado a partir dos descritores utilizados nas publicações elegíveis. 
operatório foi marcado (se aplicável) e revisará, verbalmente, com a equipe de anestesia o risco de perda sanguínea, dificuldades com vias aéreas, alergias, bem como disponibilidade e segurança dos equipamentos anestésicos e medicamentos ${ }^{1,12,15}$.

Todas essas ações visam à melhoria nos padrões de cuidado mediante comunicação interprofissional segura e redução de danos físicos e psicológicos ao paciente nesse ambiente de saúde.

Assim, a equipe de enfermagem tem papel essencial na adesão à lista de verificação, pois se responsabiliza pela qualificação, comunicação e capacitação profissional, com vistas à melhoria do serviço e à redução de EA.

Para isso, a equipe precisa estar engajada e comprometida individual e coletivamente, de modo a compreender a importância e a necessidade do uso da lista de verificação e, a posteriori, atribuir ações corretivas por meio de indicadores seguros. A enfermagem responsabiliza-se pelo planejamento de suas ações, pelo dimensionamento de pessoal, preconizado pela Portaria $n^{\circ}$ 543/ 2017 do Conselho Federal de Enfermagem (Cofen) e pelas capacitações e pelos treinamentos das equipes de trabalho no cuidado seguro 5 .

A educação permanente é um instrumento de gestão do cuidado em enfermagem, pois compreende as potencialidades $\mathrm{e}$ dificuldades existentes no cotidiano do profissional ${ }^{16}$. Além disso, favorece a comunicação interpessoal, pautada na troca de saberes, na horizontalidade, na escuta e na receptividade de novas ideias, conduzindo toda a equipe à compreensão e ao entendimento ${ }^{17}$.

Dessa forma, a segurança do paciente deve ser monitorada e mensurada por meio de uma definição clara e precisa do problema clínico a investigar no serviço de saúde. Em um estudo realizado no Texas, nos Estados Unidos, pesquisadores elaboraram o Safety Attitudes Questionnaire (SAQ), que mensura o clima de segurança percebido pelos profissionais de saúde no centro cirúrgico (CC). Esse instrumento possibilitou obter dados sobre a perspectiva dos profissionais de saúde e gestores clínicos e administrativos, propondo intervenções, direções e alternativas para a promoção na segurança do paciente ${ }^{18,19}$. A partir da representação dos valores e das ações da instituição, no que diz respeito às percepções dos profissionais sobre o gerenciamento da segurança na instituição, é possível identificar e administrar a segurança do paciente no ambiente cirúrgico.

\section{CONSIDERAÇÕES FINAIS}

A cirurgia segura está sendo cada vez mais elucidada nos serviços de saúde e discutida entre os pares. A utilização de checklist está sendo implementada por diversas instituições de saúde, conforme seu valor e sua cultura organizacional, o que resulta em benefício tanto para os profissionais quanto para os pacientes. De fato, com a implementação de checklists voltados para a cirurgia segura na década passada e com a análise, presente, dos indicadores clínicos confiáveis dos eventos adversos, podese obter dados de intervenção mais confiáveis e sistemáticos dos modelos de alto nível de organização que demonstrem melhoria na segurança dos serviços de saúde como um todo. Há uma década compreendia-se a fenomenologia dos danos e suas causas, porém, hoje não há fortes evidências que indiquem notória melhoria na segurança do paciente em todos os eixos preconizados pela OMS e, também, em toda estrutura organizacional dos serviços de saúde.

Destaca-se, ainda, que a liderança participativa da enfermagem por meio da comunicação entre a equipe, com o paciente, com os familiares e com os gestores hospitalares promove cuidado centrado no paciente, de forma continuada e segura.

\section{REFERÊNCIAS}

1. Brasil. Ministério da Saúde (MS). Segundo desafio global para a segurança do paciente: cirurgias seguras salvam vidas. Manual. Rio de Janeiro: Organização Pan-Americana da Saúde; Ministério da Saúde; Agência Nacional de Vigilância Sanitária; 2009.

2. Carvalho PA, Göttems LBD, Pires MRGM, Oliveira MLC. Cultura de segurança no centro cirúrgico de um hospital público, na percepção dos profissionais de saúde. Rev Latino-Am Enferm. 2015; 23(6):10418. DOI: 10.1590/0104-1169.0669.2647

3. Brasil. Ministério da Saúde (MS). Portaria n. 529, de 1 de abril de 2013. Institui o Programa Nacional de Segurança do Paciente (PNSP). Brasília: Ministério da Saúde; 2013.
4. Zaheer S, Ginsburg, Chuang YT, Grace SL. Patient safety climate (PSC) perceptions of frontline staff in acute care hospitals. Health Care Manage Rev. 2015;40(1):13-23. DOI: 10.1097/HMR.0000000000000005

5. Cavalcante AC, Rocha RC, Nogueira LT, Avelino FVSD, Rocha SS. Cuidado seguro ao paciente: contribuições da enfermagem. Rev Cubana Enfermería [Internet]. 2015 [citado 23 set. 2017];31(4). Disponível em: http://www.revenfermeria.sld.cu/index.php/enf/ article/view/907

6. Souza RFF, Silva LD. Estudo exploratório das iniciativas acerca da segurança do paciente em hospitais do Rio de Janeiro. Rev Enferm UERJ. 2014;22(1):22-8. 
7. Mendes KDS, Silveira RCCP, Galvão CM. Revisão integrativa: método de pesquisa para a incorporação de evidências na saúde e na enfermagem. Rev Texto Contexto Enferm. 2008;17(4):758-64.

8. Souza NA, Boruchovitch E. Mapas conceituais: estratégia de ensino/ aprendizagem e ferramenta avaliativa. Educrev [Internet]. 2010 [citado 17 jul. 2017];26(3):195-218. Disponível em: http://www.scielo.br/pdf/edur/v26n3/ v26n3a10.pdf. DOl: http://dx.doi.org/10.1590/S0102-46982010000300010

9. Galvão TF, Pansani TSA. Principais itens para relatar revisões sistemáticas e meta-análises: a recomendação PRISMA - tradução. Epidemiol Serv Saúde [Internet]. 2015 [citado 28 jul. 2017];24(2):335-42. Disponível em: http://www.scielo.br/pdf/ress/v24n2/2237-9622-ress-24-02-00335. pdf. DOI: http://dx.doi.org/10.5123/S1679-49742015000200017

10. Agência Nacional de Vigilância Sanitária (Anvisa). Manual Cirurgias Seguras Salvam Vidas. Aliança Mundial para Segurança do Paciente. 2008 [citado 19 jul. 2017]. Disponível em: http://bvsms.saude.gov.br/bvs/publicacoes/ seguranca_paciente_cirurgias_seguras_salvam_vidas.pdf

11. Araújo MPS, Oliveira AC. Quais mudanças poderão ocorrer na assistência cirúrgica após implantação dos núcleos de Segurança do Paciente? Rev Enferm Cent 0 Min [Internet]. 2015 [citado 19 jul. 2017];5(1):1542-51. Disponível em: http://www.seer.ufsj.edu.br/index.php/recom/article/ view/807/844. DOI: http://dx.doi.org/10.19175/recom.v0i0.807

12. Pancieri AP, Carvalho R, Braga EM. Aplicação do checklist para cirurgia segura: relato de experiência. Rev SOBECC. 2014;19(1):2633. DOI: http://dx.doi.org/10.4322/sobecc.2014.006

13. Pancieri AP, Santos BP, Avila MAG, Braga EM. Safe surgery checklist: analysis of the safety and communication of teams from a teaching hospital. Rev Gaúcha Enferm. 2013;34(1):71-8. DOI: http://dx.doi. org/10.1590/S1983-14472013000100009
14. Santos CM, Caregnato RCA, Moraes CS. Equipe cirúrgica: adesão à meta 1 da cirurgia segura. Rev SOBECC [Internet]. 2013 [citado 19 jul. 2017];18(4):47-56. Disponível em: http://www.sobecc.org. br/arquivos/artigos/2014/pdfs/revisao-de-leitura/Ano18_n4_out_ dezet2013-9.pdf

15. Gomes CDPP, Santos AA, Machado ME, Treviso P. Percepção de uma equipe de enfermagem sobre a utilização do checklist cirúrgico. Rev SOBECC. 2016;21(3):140-5. DOI: http://dx.doi.org/10.5327/ Z1414-4425201600030004

16. Koerich C, Erdmann AL. Significados atribuídos pela equipe de enfermagem sobre educação permanente em uma instituição cardiovascular. Rev Rene [Internet]. 2016 [citado 28 ago. 2017];17(1):93-102. Disponível em: http://www.revistarene. ufc.br/revista/index.php/revista/article/view/2223/pdf. DOI: 10.15253/2175-6783.2016000100013

17. Coriolano-Marinus MWL, Queiroga BAM, Ruiz-Moreno L, Lima LS. Comunicação nas práticas em saúde: revisão integrativa da literatura. Rev Saúde Sociedade [Internet]. 2014 [citado 28 ago. 2017];23(4):1356-69. Disponível em: https://www.revistas.usp.br/ sausoc/article/view/104305/102952. DOl: http://dx.doi.org/10.1590/ S0104-12902014000400019

18. Elias ACGP, Schmidt DRC, Yonekura SCI, Dias AO, Ursi ES, Silva RPJ, et al. Avaliação da adesão ao checklist de cirurgia segura em hospital universitário público. Rev SOBECC. 2015;20(3):128-33.

19. Moura MLO, Mendes W. Avaliação de eventos adversos cirúrgicos em hospitais do Rio de Janeiro. Rev Bras Epidemiol [Internet]. 2012 [citado 17 jul. 2017];15(3):523-35. Disponível em: http://www. scielo.br/pdf/rbepid/v15n3/07.pdf. DOl: http://dx.doi.org/10.1590/ S1415-790X2012000300007 